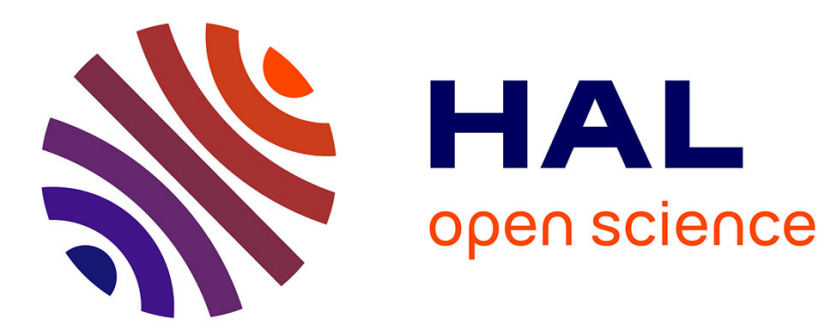

\title{
Utilisation d'un maser à ammoniac en résonance magnétique électronique (RPE)
}

\author{
J. Hardin, J. Uebersfeld
}

\section{To cite this version:}

J. Hardin, J. Uebersfeld. Utilisation d'un maser à ammoniac en résonance magnétique électronique (RPE). Revue de Physique Appliquée, 1971, 6 (2), pp.169-176. 10.1051/rphysap:0197100602016900 . jpa-00243517

\section{HAL Id: jpa-00243517 https://hal.science/jpa-00243517}

Submitted on 1 Jan 1971

HAL is a multi-disciplinary open access archive for the deposit and dissemination of scientific research documents, whether they are published or not. The documents may come from teaching and research institutions in France or abroad, or from public or private research centers.
L'archive ouverte pluridisciplinaire HAL, est destinée au dépôt et à la diffusion de documents scientifiques de niveau recherche, publiés ou non, émanant des établissements d'enseignement et de recherche français ou étrangers, des laboratoires publics ou privés. 


\title{
UTILISATION D'UN MASER A AMMONIAC EN RÉSONANCE MAGNÉTIQUE ÉLECTRONIQUE (RPE) (*)
}

\author{
par J. HARDIN et J. UEBERSFELD
}

Laboratoire de l'Horloge Atomique du C. N. R. S., 25, Besançon

Laboratoire de Résonance Magnétique, Faculté des Sciences de Paris

\begin{abstract}
Résumé. - On a construit un maser à ammoniac qui oscille avec une cavité résonnante constituée par deux résonateurs couplés. Le premier résonateur reçoit le jet de ( molécules actives ), le second est placé dans le champ magnétique et contient l'échantillon que l'on désire étudier par résonance paramagnétique électronique (RPE).

Ce maser oscillateur se comporte vis-à-vis du phénomène de RPE comme un ( oscillateur marginal ) utilisé en résonance magnétique nucléaire. On a étudié la réponse du maser à un phénomène de RPE en fonction du niveau d'oscillation et de la fréquence de modulation. L'étude du bruit de l'oscillateur, d'autre part, a permis de montrer que le bruit de scintillation est négligeable et que la sensibilité est seulement limitée par le bruit thermique dans la cavité. La sensibilité expérimentale mesurée est effectivement celle d'un spectromètre idéal, c'est-à-dire celle d'un spectromètre classique avec un récepteur parfait.
\end{abstract}

Il est possible d'exciter l'échantillon avec une puissance aussi faible que $10^{-12} \mathrm{~W}$.

Abstract. - An ammoniac maser which oscillates with a resonant cavity constituted by two coupled resonators has been built. The first resonator receives the beam of active molecules, the second one is set in the magnetic field and contains the sample we want to study by electronic paramagnetic resonance.

This maser oscillator behaves towards the phenomenon of RPE like a ( marginal oscillator ) used in nuclear magnetic resonance. We have studied the response of the maser depending on the oscillation level and the modulation frequency. The study of the oscillator noise, on the other hand enabled us to show that the ( flicker ) noise is negligible and that the sensitivity is only limited by the thermal noise in the cavity. The measured experimental sensitivity is in fact that of an ideal spectrometer, that is to say that of a classical spectrometer using a perfect receiver.

The sample may be excited to a power level as low as $10^{-12} \mathrm{~W}$.

Introduction. - Pour situer cette étude dans l'ensemble des travaux tendant à améliorer la sensibilité des spectromètres hertziens, il convient de rappeler brièvement les caractéristiques des spectromètres conventionnels dont le schéma de principe est donné sur la figure 1. On y reconnaît l'oscillateur qui constitue la source d'excitation, la cellule de mesure contenant le corps à étudier qui, en ondes centimétriques, est géné-

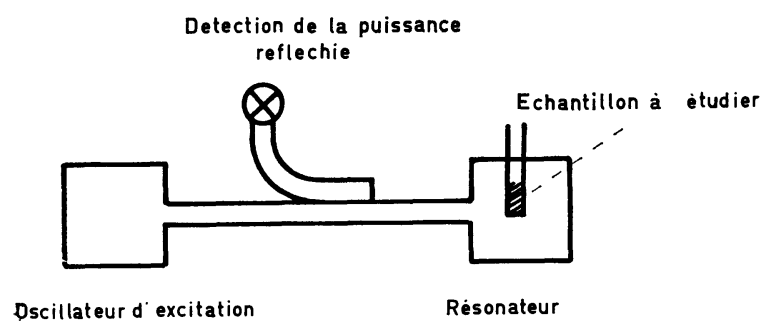

Fig. 1. - Schéma de principe d'un spectromètre classique.

(*) Cette étude constitue une partie du mémoire de thèse de J. Hardin. No C. N. R. S., AO. 3779. ralement une cavité résonnante. Un récepteur permet de mettre en évidence la variation d'énergie réfléchie lors du passage à la résonance de l'échantillon. La sensibilité d'un tel spectromètre s'exprime par la relation

$$
\chi_{\min }^{\prime \prime}=\frac{2}{\eta Q_{0}} \sqrt{\frac{F k T \Delta f}{P}}
$$

où $\chi_{\min }^{\prime \prime}$ est l'absorption minimum détectable ; $Q_{0}$ le coefficient de qualité du résonateur, $\eta$ son coefficient de remplissage ; $P$ la puissance d'excitation ; $F$ le facteur de bruit du récepteur ; $\Delta f$ la bande passante de détection ; $k$ la constante de Boltzmann ; $T$ la température absolue. Cette relation ne tient pas compte du bruit de l'oscillateur d'excitation.

L'utilisation des masers pour améliorer la sensibilité des spectromètres hertziens a consisté à employer des masers amplificateurs :

- soit, comme étage préamplificateur à faible bruit, dans des montages classiques, afin d'améliorer le facteur de bruit $F[1]$;

- soit, sur la suggestion de Townes [2], comme « multiplicateur de facteur de qualité ». 
Nous avons employé le maser oscillateur dans un montage de type autodyne largement répandu dans le domaine des radiofréquences, de quelques mégahertz à quelques centaines de mégahertz pour l'étude des raies d'absorption.

La caractéristique de ce type de spectromètre est que la cellule de mesure fait partie du circuit d'accord de l'oscillateur, le phénomène physique étant mis en évidence par les variations du niveau d'oscillation qu'il entraîne.

Une propriété de ce type de montage est sa très grande sensibilité théorique. En se rapprochant du seuil d'oscillation, on peut rendre le bruit propre de l'oscillateur supérieur au bruit du récepteur et comme le «signal » comportant l'information physique augmente généralement de la même façon, on peut ainsi s'affranchir du bruit du récepteur. Toutefois cet avantage est le plus souvent illusoire, le bruit de scintillation des oscillateurs électroniques étant par trop élevé et finalement la sensibilité de ce type de montage n'est pas supérieur aux autres.

Mais l'existence d'oscillateurs quantiques, tel le maser à ammoniac, où le bruit de scintillation, comme nous le montrerons, est quasiment négligeable, permet d'espérer la réalisation d'un spectromètre atteignant la sensibilité limite théorique «idéale », c'est-à-dire conforme à la formule (1) où $F=1$. Il est clair que la réalisation d'un tel spectromètre passe d'une part par la construction d'un maser de grande sûreté de fonctionnement et de réglage aisé, d'autre part par l'étude détaillée de ses performances comme générateur hyperfréquence.

I. Le maser considère comme un générateur hyperfréquence. - La construction du maser et des dispositifs associés ayant été décrits par ailleurs [3], [4] nous rappellerons seulement le principe de fonctionnement, les hypothèses de calculs et les principaux résultats de l'étude du maser considéré comme un oscillateur radioélectrique classique.

1. Principe de fonctionnement. - Il repose sur le phénomène d'émission stimulée. Un jet de molécules d'ammoniac préalablement enrichi en molécules dans l'état supérieur d'énergie, traverse une cavité résonnante dont la fréquence de résonance est réglée au voisinage de la fréquence de la transition qui est de l'ordre de $23870 \mathrm{MHz}$. Dès que la puissance fournie par les molécules compense les pertes dans la cavité et dans les lignes de sortie, un régime d'oscillation s'établit. L'inversion de population nécessaire est réalisée par un séparateur électrostatique appelé aussi focaliseur.

La figure 2 montre le schéma de l'appareil. On y reconnaît le gicleur $(a)$ par où l'ammoniac est introduit sous forme de jet dans l'enceinte où règne une basse pression, de l'ordre de $10^{-7} \mathrm{~mm}$ de $\mathrm{Hg}$. La pression en amont du gicleur est contrôlée par une vanne $(c)$ et mesurée sur une jauge de Pirani $(d)$. Le focaliseur $(e)$ est formé de huit barres portées alternativement à des potentiels $\mathrm{O}$ et $\mathrm{V}$. Il défocalise les molécules dans l'état inférieur qui viennent se fixer sur le piège à azote liquide $(f)$ entourant le système. Un piège terminal $(h)$ capte les molécules ayant traversé la cavité $(g)$. Cette cavité est munie de deux lignes de sortie, l'une (1) analogue à celle des masers utilisés comme étalon de

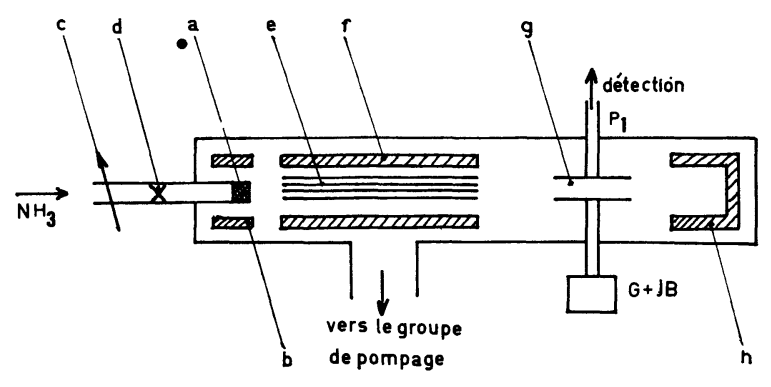

Fig. 2. - Schéma de principe du maser.

fréquence ; l'autre (2) comportant une charge variable étalonnée permettant de faire varier de quantités connues le facteur de qualité et la fréquence de résonance de la cavité.

2. Hypothèses de calcul. - Nous avons employé le formalisme développé par Slater [5] dans l'étude des klystrons. Il consiste à écrire, pour des plans de référence choisis, la condition d'oscillation à partir de l'admittance active d'un faisceau d'électrons et des paramètres radioélectriques $\mathrm{du}$ résonateur.

$$
\begin{aligned}
-\frac{g_{m}+j b_{m}}{C \omega_{\mathrm{c}}} & =\frac{1}{Q_{0}}+\frac{1}{Q_{1}}+\frac{G}{Q_{2}}+j\left(\frac{\omega}{\omega_{\mathrm{c}}}-\frac{\omega_{\mathrm{c}}}{\omega}+\frac{B}{2 Q_{2}}\right) \\
& =\frac{1}{Q_{T}}+j\left(\frac{\omega}{\omega_{\mathrm{c}}}-\frac{\omega_{\mathrm{c}}}{\omega}+\frac{B}{2 Q_{2}}\right) .
\end{aligned}
$$

Dans notre problème, le rôle de l'admittance électronique est joué par la quantité $g_{m}+j b_{m}$ que nous appelons par analogie l'admittance moléculaire du jet. $C$ est un facteur qui dépend de la géométrie de la cavité et qui a les dimensions d'une capacité. Dans les relations relatives aux klystrons, c'est la capacité du « gap ». $Q_{0}$ est le coefficient de qualité à vide de la cavité ; $Q_{1}$ et $Q_{2}$, les coefficients relatifs aux lignes (1) et (2); $\omega_{c}$ la fréquence angulaire de résonance de la cavité ; $G$ et $B$ les parties réelle et imaginaire de l'admittance réduite de la charge variable.

Les expressions de $g_{m}$ et $b_{m}$ sont obtenues à l'aide de la théorie de Shimoda, Wang et Townes (SWT) [6], développée pour le maser à ammoniac dans l'hypothèse où un résonateur dans lequel la répartition du champ électrique est uniforme, est traversẻ par un jet de molécules monocinétique dont les trajectoires sont parallèles à l'axe de la cavité. La cavité employée, qui résonne dans le mode TM 010 , réalise approximativement la condition d'uniformité du champ. 


$$
\begin{gathered}
g_{m}=\frac{-\mu^{2}}{2 \hbar v^{2}} n \omega \frac{\sin ^{2} \Theta}{\Theta^{2}} \\
b_{m}=\frac{\mu^{2}}{2 \hbar v^{2}} n \omega \frac{1}{2\left(\frac{v}{L}\right)} \frac{\omega-\omega_{r}}{\Theta^{2}}\left[1-\frac{\sin 2 \Theta}{2 \Theta}\right] \\
\Theta=\left[\left(\omega-\omega_{r}\right)^{2}+\left(\frac{\mu E}{\hbar}\right)^{2}\right]^{1 / 2} \frac{L}{2 v}, \quad \theta=\frac{\mu E}{\hbar} \cdot \frac{L}{2 v} .
\end{gathered}
$$

Dans ces relations :

$\mu$ est le moment dipolaire de la transition ; $v$ la vitesse des molécules ; $n$ leur débit ; $L$ la longueur de la cavité ; $\omega_{\boldsymbol{r}}$ la fréquence de transition ; $E$ l'amplitude du champ électrique.

La résolution des 2 équations, tirées de la condition d'oscillation, nous conduit au régime stationnaire d'oscillation. L'expression générale donnant la fréquence d'oscillation est :

$\omega-\omega_{r}=\frac{Q_{T}}{Q_{r}} \cdot \frac{1-\cos 2 \Theta}{\pi\left(1-\frac{\sin 2 \Theta}{2 \Theta}\right)}\left[\frac{\omega-\omega_{c}}{\omega_{c}}-\frac{B}{2 Q_{2}}\right]$.

Dans cette formule nous avons introduit $Q_{r}=f_{r} \cdot(L / v)$ que l'on appelle le facteur de qualité de la raie et qui est lié à la largeur en fréquence de la raie moléculaire. $Q_{r}$ est de l'ordre de $4 \times 10^{6}$; nous le considérons comme une constante : c'est notre première constante d'appareillage.

Ces expressions sont des expressions générales qui se simplifient pour $\omega$ très voisin de $\omega_{r}$.

a) La puissance dissipée est proportionnelle à $1 / Q_{T}$ et nous pouvons définir de la même façon un facteur $1 / Q_{m}$ qui est proportionnel à la puissance $P_{m}$ fournie par le jet de molécules

$$
P_{m}=\frac{\omega W}{Q_{m}}
$$

si $W$ est l'énergie emmagasinée. Dans ce cas, la condition d'oscillation s'écrit

$$
\frac{1}{Q_{T}}=\frac{1}{Q_{m_{0}}} \frac{\sin ^{2} \theta}{\theta^{2}}
$$

avec

$$
\frac{1}{Q_{m_{0}}}=\frac{1}{\varepsilon_{0} A} \frac{\mu^{2}}{2 \hbar v^{2}}
$$

b) Si la répartition du champ dans le résonateur est uniforme, l'énergie emmagasinée est liée au paramètre $\theta$ par la relation

$$
W=\theta^{2} W_{c} \text { avec } W_{c}=\frac{\varepsilon_{0} A v^{2} \hbar^{2}}{2 \pi^{2} \mu^{2} L} .
$$

Ce paramètre $W_{c}$, introduit par S.W.T., est dans l'hypothèse du jet monocinétique une constante et vaut dans notre cas $5 \times 10^{-18} \mathrm{~J}$ : ce sera notre seconde constante d'appareillage. c) La puissance émise dans les guides (1) et (2) s'exprime par :

$$
\begin{aligned}
& P_{1}=P_{0} \theta^{2} \\
& P_{2}=P_{0} G \frac{Q_{1}}{Q_{2}} \theta^{2}
\end{aligned}
$$

en posant :

$$
P_{0}=\frac{\omega W_{c}}{Q_{1}}
$$

Cette quantité $P_{0}$ qui joue un rôle particulier, puisque dans l'emploi en spectromètre, la puissante est détectée dans la ligne (1), est notre $3^{\mathrm{e}}$ constante d'appareillage ; elle vaut pour le couplage considéré $9 \times 10^{-11} \mathrm{~W}$.

Le système de mesure de la puissance ayant été soigneusement étalonné, la mesure de $P$ nous conduit immédiatement à la valeur de $\theta$.

3. Résultats principaux. - L'étude radioélectrique du maser montre :

- que le maser est un oscillateur de type marginal, de grande dynamique (30 db), de puissance d'émission faible $\left(3 \times 10^{-10} \mathrm{~W}\right.$ maximum) et de grande stabilité de fréquence par rapport aux paramètres du jet;

- qu'une très faible variation du coefficient de qualité du résonateur entraîne de fortes variations $d u$ niveau d'oscillation ;

- que, du fait de l'étroitesse de la réponse en fréquence du système moléculaire $(7 \mathrm{KHz})$, les diagrammes de performances [7] permettent de résoudre sans ambiguïté le problème du maser oscillant sur deux cavités couplées par un tronçon de ligne. Dans ce montage, la cavité 1 est la cavité traversée par le jet, la charge variable est remplacée par la charge réactive formée par la ligne terminée par la cavité 2 (de coefficient de qualité $Q_{1}^{\prime}, Q_{0}^{\prime}$ ) (figure 3 ).

Dans le cas du « couplage coïncidant ", c'est-à-dire si la longueur de la ligne est $n / 2 . \lambda g, \lambda g$ étant la longueur d'onde guidée, une faible diminution de $Q_{0}^{\prime}$ entraîne une baisse du niveau de l'oscillateur.

II. Le maser appliqué à l'étude de l'absorption paramagnétique. - Utiliser le maser muni de ses deux cavités couplées par un tronçon de ligne en spectromètre pour R. P. E. va consister à placer dans la cavité 2 l'échantillon du corps à étudier.

Le phénomène de résonance magnétique va se traduire macroscopiquement par une modification des caractéristiques de la $2^{\mathrm{e}}$ cavité liée à la susceptibilité complexe de l'échantillon $\chi=\chi^{\prime}-j \chi^{\prime \prime}$ par

$$
\Delta\left(\frac{1}{Q_{0}^{\prime}}\right)=\eta_{0}^{\prime} \chi^{\prime \prime}
$$

pour la variation du coefficient de qualité

$$
\frac{\Delta f}{f}=\frac{\eta_{0}^{\prime}}{2} \chi^{\prime}
$$

pour la variation relative de sa fréquence,

$\eta_{0}^{\prime}$ étant le coefficient de remplissage de la cavité 2 , analogue à celui qui intervient dans la formule $\mathrm{I}$. 
D'après les résultats précédents, la modification de $Q_{0}^{\prime}$ va entraîner une diminution du niveau de l'oscillateur et une légère variation de sa fréquence. On a bien à faire à un spectromètre " autodyne » du type décrit dans l'introduction où la cellule de mesure fait partie intégrante du circuit d'accord de l'oscillateur. La variation de fréquence sera très faible étant donné la petitesse du facteur d'entraînement $Q_{T} / Q_{r}$ et de toute manière, les variations $\mathrm{du}$ niveau que nous observerons ne seront liées qu'au phénomène d'absorption.

La figure 3 montre le schéma de principe du spectromètre. On y reconnaît le maser et la cavité 1 , la cavité 2

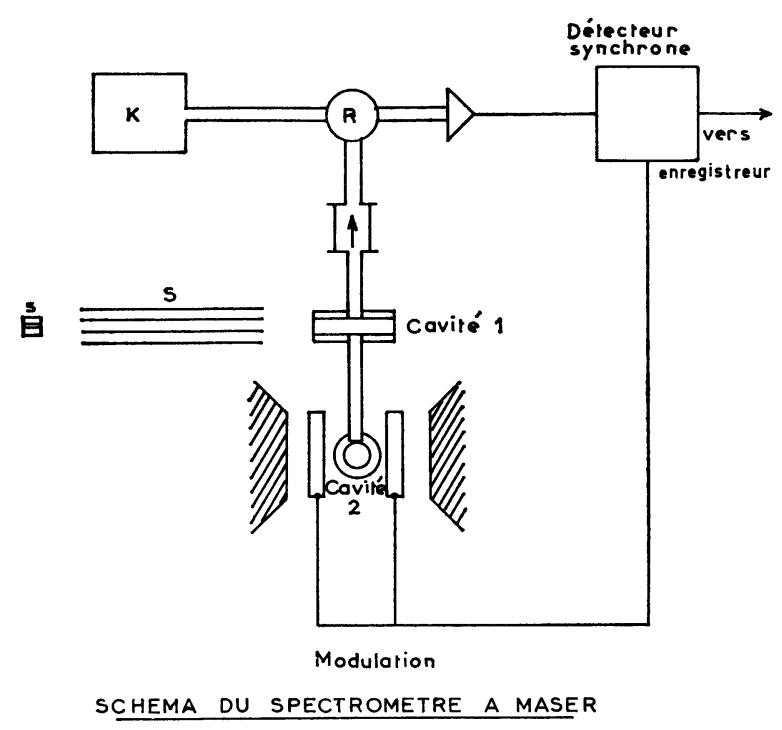

FIG. 3. - Schéma de principe du spectromètre à̃

entre les pôles de l'électro-aimant et les bobines de modulation permettant de moduler en basse fréquence le phénomène d'absorption. A la sortie du récepteur, on fait une détection synchrone des variations du niveau du maser, à la fréquence de modulation optimale. Pour la déterminer, il faut connaître le temps de retour à l'équilibre de l'oscillateur légèrement écarté de son niveau d'oscillation, c'est-à-dire déterminer ce qu'on appelle la bande passante basse fréquence de l'oscillateur. Pour la calculer il faut posséder l'équation différentielle régissant le comportement de l'oscillateur, au voisinage d'un état d'équilibre et pour ce faire, il faut choisir un schéma radioélectrique équivalent.

Ce schéma équivalent doit tenir compte d'une caractéristique importante du maser. La non-linéarité conduisant à la limitation de l'amplitude dépend de l'amplitude du champ électrique et non de sa valeur instantanée : par nature le maser à ammoniac est un oscillateur à filtre d'enveloppe.

1. EQUATION PHÉNOMÉNOLOGIQUE RÉGISSANT LE COMPORTEMENT DU MASER. - - a) Le schéma équivalent : une discussion détaillée de ce schéma [8] conduit à l'équation suivante :

$$
\frac{\mathrm{d}^{2} e}{\mathrm{~d} t^{2}}+\frac{\omega_{r}}{Q_{r}}\left(1-\frac{Q_{T}}{Q_{m_{0}}} \frac{\sin ^{2} \theta}{\theta^{2}}\right) \frac{\mathrm{d} e}{\mathrm{~d} t}+\omega_{r}^{2} e=0
$$

où $e$ est la valeur instantanée du champ électrique régnant dans la cavité $1 . Q_{r}$ est le facteur de qualité moléculaire et qui vaut environ 1000 fois le coefficient de qualité $Q_{T}$ du circuit de réaction que représente la cavité. Ceci différencie le maser des oscillateurs radioélectriques classiques où le « circuit amplificateur »a une bande passante beaucoup plus grande que le résonateur.

b) Réponse à une faible modulation du $Q_{0}^{\prime}$ de la cavité 2 . Bande passante "basse fréquence». Considérons alors la réponse du maser à une faible perturbation de $Q_{0}^{\prime}$, à la fréquence $\Omega / 2 \pi$, au voisinage de l'amplitude du régime $\theta_{0}, \Omega$ étant très petit devant $\omega_{r}$ $\left(\omega_{r} \approx 10^{9} \Omega\right)$. Nous avons résolu cette équation par la méthode de la moyenne de Haag [9], [10] et l'on trouve pour le «signal » à la fréquence $\Omega$, à la sortie du récepteur, caractérisé par son gain $K$

$$
\begin{aligned}
\Delta s= & K \frac{\sqrt{P_{0}}}{2} \cdot \frac{\omega_{r} Q_{1}^{\prime} Q_{T}}{Q_{r} Q_{2}} \cdot \theta_{0} \times \\
& \times\left\{\left[\frac{\omega_{r}}{Q_{r}}\left(1-\theta_{0} \operatorname{cotg} \theta_{0}\right)\right]^{2}+\Omega^{2}\right\}^{-1 / 2} \cdot \Delta\left(\frac{1}{Q_{0}^{\prime}}\right)
\end{aligned}
$$

$Q_{1}^{\prime}$ et $Q_{0}^{\prime}$ apparaissant du fait que l'on a explicité dans les calculs la dépendance de $Q_{T}$ en fonction des paramètres de la cavité 2 .

On retrouve ici un résultat classique [11] : l'oscillateur haute fréquence, soumis à une perturbation périodique, en basse fréquence de son circuit d'accord, se conduit vis-à-vis de cette modulation comme un amplificateur basse fréquence dont le gain et la bande passante dépendent du niveau d'oscillation. La bande passante pour le niveau $\theta_{0}$ est donnée par la relation

$$
\Delta B=\frac{\omega_{r}}{Q_{r}}\left(1-\theta_{0} \operatorname{cotg} \theta_{0}\right) .
$$

c) Vérifications expérimentales : Nous avons mesuré la bande passante indépendamment du phénomène de R. P. E., en modulant faiblement en signaux carrés le niveau du maser à l'aide de la charge étalonnée équipée d'un système de modulation de son coefficient de réflexion.

La figure 4 montre la comparaison entre les valeurs calculées et les valeurs mesurées. La courbe en trait plein est la courbe théorique déterminée d'après la relation (5) avec $Q_{r}=4 \times 10^{6}$ et les valeurs de $\theta_{0}$ déduites de la valeur de $P$ et de $P_{0}$.

Pour les forts niveaux d'oscillation, l'accord est excellent mais on note un écart systématique pour les faibles valeurs. Une des raisons pourrait être qu'en fait le jet n'est pas monocinétique et qu'aux faibles valeurs du champ, ce sont les molécules les plus lentes qui ont la plus grande probabilité de transition. Ce qui conduit, 
macroscopiquement, à prendre un $Q_{r}$ plus grand, donc une bande passante plus étroite. Cet argument a d'ailleurs le mérite de ne pas remettre en cause le schéma équivalent mais seulement la constance d'un des paramètres macroscopiques.

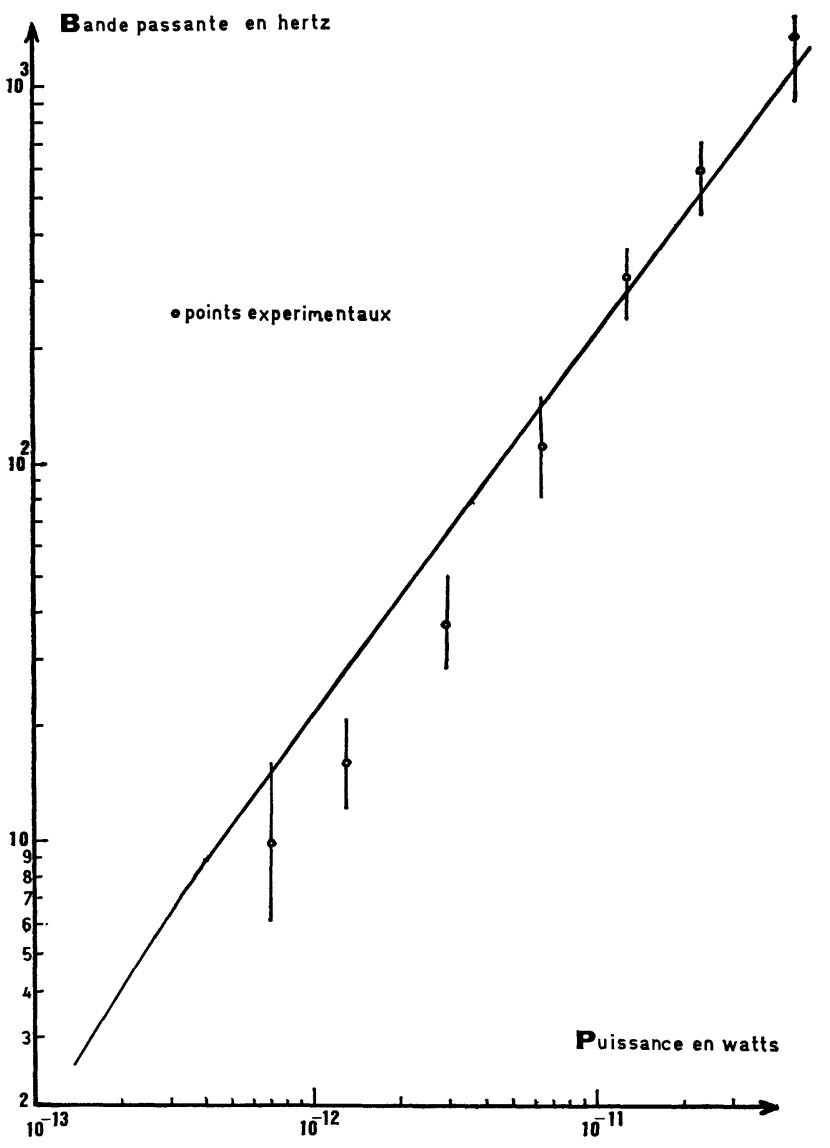

FIG. 4. - Bande passante de l'oscillateur en fonction du niveau d'oscillation.

Nous avons cherché à vérifier ensuite si la loi d'évolution du signal, en fonction de la fréquence de modulation du niveau et pour un $\Delta\left(1 / Q_{0}^{\prime}\right)$ donné, suivait la loi théorique.

La figure 5 montre les courbes théoriques et les points

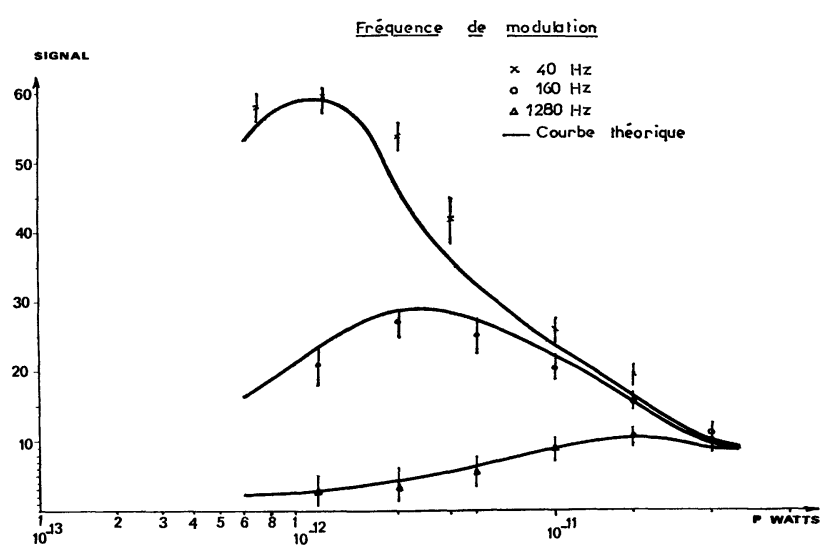

FIG. 5. - Evolution du signal en fonction du niveau d'oscillation pour différentes fréquences de modulation. expérimentaux pour 3 valeurs de la fréquence de modulation. La concentration du petit échantillon employé n'étant pas connue avec précision, nous avons fait coïncider un point expérimental avec une valeur théorique pour obtenir le facteur de proportionnalité. L'accord est bon et ce réseau illustre bien le fait que le gain basse fréquence dépend du niveau d'oscillation.

2. BRUIT DE L'OSCILlateUR. - Nous connaissons maintenant l'évolution du signal puisque

$$
\Delta\left(1 / Q_{0}^{\prime}\right)=\eta_{0}^{\prime} \chi^{\prime \prime} \text {; }
$$

pour donner la sensibilité théorique de l'appareillage, il faut comparer théoriquement le signal au bruit.

Comme nous le verrons, notre montage permet de s'affranchir du bruit du récepteur et nous allons considérer tout d'abord les sources du bruit d'amplitude de l'oscillateur. Ce sont :

- le phénomène d'émission spontanée,

- les fluctuations du nombre de molécules dans le jet ce qui correspond dans notre cas au bruit de scintillation,

- l'amplification du rayonnement thermique $d u$ résonateur par le système moléculaire.

Dans le domaine hertzien, par suite de la petitesse du quantum par rapport au quantum thermique à la température ordinaire, l'influence de l'émission spontanée est négligeable. La fluctuation du nombre de molécules, elle aussi, est négligeable par suite du grand nombre de molécules (de l'ordre de $10^{10}$ ) présentes à tout instant dans le résonateur. Néanmoins, nous avons cherché à le vérifier expérimentalement en simulant, pour différents niveaux d'oscillation, une petite variation du nombre de molécules actives. Nous avons superposé à la tension de focalisation, une faible tension sinusoïdale, d'amplitude constante et de fréquence variable et opéré une détection synchrone des variations du niveau d'oscillation.

Cette étude a permis de déterminer la sensibilité du maser aux fluctuations de tension de focalisation. Compte tenu du spectre des fluctuations d'amplitude $\mathrm{du}$ générateur électrostatique utilisé, on peut affirmer que sauf peut-être au voisinage immédiat du seuil d'oscillation, les fluctuations d'amplitude du maser ne sont pas liées à la scintillation.

Reste à étudier le bruit d'origine thermique. Pour l'étudier théoriquement, nous avons cherché la réponse $\mathrm{du}$ maser à une faible perturbation sinusoïdale de fréquence voisine de $\omega_{r}$. Nous en avons déduit une bande passante " haute fréquence ». Ayant mis en évidence un temps de retour à l'équilibre de l'oscillateur, nous écrivons que chaque composante du bruit thermique, le bruit étant représenté par ses composants de Fourier, donne lieu à une réponse de régime permanent. Ceci nous permet de déterminer le spectre théorique des fluctuations d'amplitude : c'est justement ce que nous cherchons, car nous opérons une détection synchrone du signal, modulé à la fréquence $F$; ce qu'il 
nous faut comparer au signal est le bruit à la distance $F$ de la porteuse dans la bande $\Delta f$ de détection.

Pour savoir comment intervient le bruit thermique dans l'équation différentielle, nous avons repris le schéma équivalent [8]. On trouve que le bruit thermique apparaît au second membre de l'équation par ses dérivées premières et secondes, sous la forme

$$
F(t)=\frac{\mathrm{d}^{2} e_{n}(t)}{\mathrm{d} t^{2}}+\frac{\omega_{r}}{Q_{r}} \frac{\mathrm{d} e_{n}(t)}{\mathrm{d} t}+\omega_{r}^{2} e_{n}(t) .
$$

En vertu des résultats sur la réponse du maser à une excitation à la fréquence $\omega$, telle que $\omega-\omega_{r}=\Omega$, la composante de la modulation de l'amplitude du champ électrique $E_{n}(\Omega)$ s'exprime par la relation :

$$
E_{n}(\Omega)=\frac{e_{n}(\Omega)\left[\Omega^{2}+\left(\frac{\omega_{r}}{2 Q_{r}}\right)^{2}\right]^{1 / 2}}{\left\{\left[\frac{\omega_{r}}{Q_{r}}\left(1-\theta_{0} \operatorname{cotg} \theta_{0}\right)\right]^{2}+\Omega^{2}\right\}^{1 / 2}}
$$

où $e_{n}(\Omega)$ représente la composante du bruit thermique $\mathrm{du}$ résonateur.

On retrouve un résultat classique : vu la petitesse de $\Omega$ devant $\omega_{r}$, le numérateur est une constante, la bande passante " haute fréquence " est la même que la bande passante «basse fréquence ».

Dans le cas où $\Omega \ll \Delta B$, c'est-à-dire pour des composantes dont l'écart à la fréquence centrale $\Omega$ est inférieur à la bande passante de l'oscillateur pour le niveau considéré, $E_{n}(\Omega)$ s'exprime par la relation

$$
E_{n}(\Omega)=\frac{e_{n}(\Omega)}{2}\left(1-\theta_{0} \operatorname{cotg} \theta_{0}\right)^{-1}
$$

Si $\Omega \gg \omega_{r} / Q_{r}$, c'est-à-dire pour des composantes qui tombent à l'extérieur de la réponse moléculaire $E_{n}(\Omega) \approx e_{n}(\Omega)$ le maser n'amplifie pas ces composantes. Le bruit thermique est alors pour l'oscillateur un bruit additif. Ce sont les résultats trouvés par Townes par une autre méthode. On peut exprimer simplement $e_{n}(\Omega)$ en considérant que pour le faible intervalle spectral considéré, le bruit thermique peut être avec une bonne approximation, considéré comme un bruit blanc. Dans ce cas, en tenant compte des couplages et $\mathrm{du}$ facteur de bruit du récepteur, la composante de la tension de bruit à la sortie du récepteur s'exprime par :

$$
\begin{aligned}
& \Delta s_{\beta}(\Omega)=K \sqrt{k T \Delta f} \times \\
& \times\left[\frac{\Omega^{2}+\left(\frac{\omega_{r}}{2 Q_{r}}\right)^{2}}{\left[\frac{\omega_{r}}{Q_{r}}\left(1-\theta_{0} \operatorname{cotg} \theta_{0}\right)\right]^{2}+\Omega^{2}} 4 \frac{Q_{1}}{Q_{T}}+F\right]^{1 / 2} .
\end{aligned}
$$

Nous avons tabulé cette expression pour différents niveaux et différents $\Omega$, et nous l'avons comparé au spectre expérimental. Sur la figure 6, les courbes théoriques sont en trait plein, les courbes expérimentales en pointillé, les barres verticales sur la partie gauche indiquent l'incertitude de mesure. On voit que l'accord est bon et qu'il existe toute une zone de fonctionnement où le bruit de l'oscillateur est beaucoup plus grand que le bruit du récepteur.

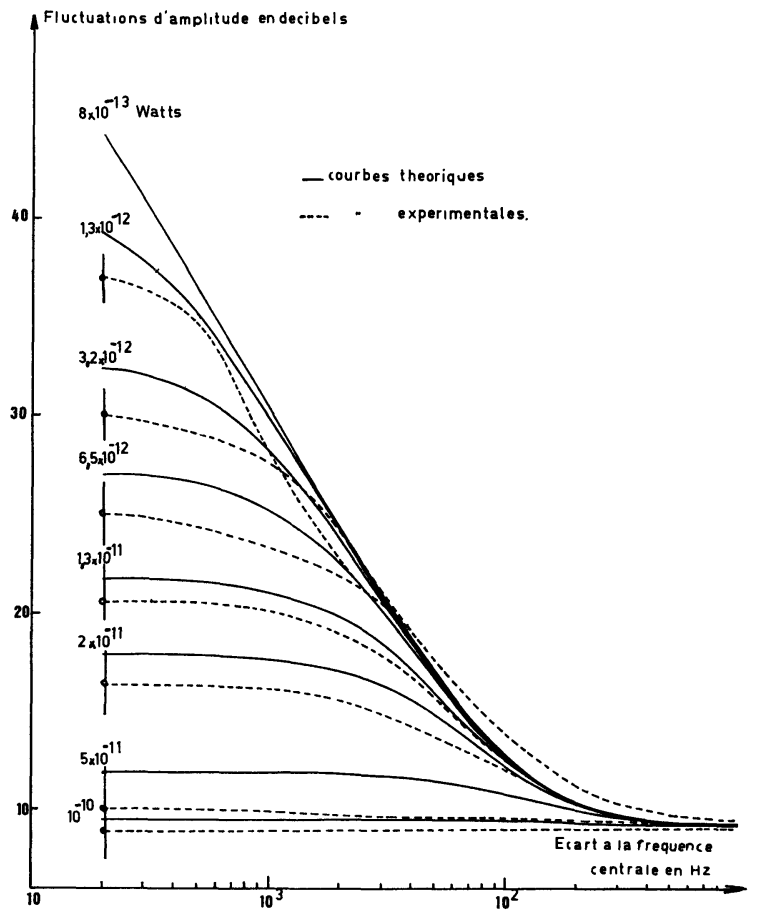

Fig. 6. - Spectre de fluctuations d'amplitude du maser.

3. Sensibilité du SPeCtromètre. - En se plaçant à un niveau d'oscillation, tel que $F$ soit négligeable devant le premier terme et à des fréquences de modulation du signal tel que $\Omega$ soit bien inférieur à la bande passante de l'oscillateur pour le niveau considéré, on peut exprimer à l'aide des formules (4) et (6) la sensibilité du spectromètre par la relation

$$
\chi_{\text {min }}^{\prime \prime}=\frac{2}{\eta_{\mathrm{e}}^{\prime} Q_{0}^{\prime}} \sqrt{\frac{k T \Delta f}{P_{u}}}
$$

$\eta_{e}^{\prime}$ est le coefficient de remplissage effectif, relatif au système des deux cavités couplées et vaut

$$
\eta_{\mathrm{e}}^{\prime}=\eta_{0}^{\prime}\left[1+\frac{Q_{2}}{Q_{1}} \cdot Q_{0}^{\prime}\left(\frac{1}{Q_{0}}+\frac{1}{Q_{1}}\right)\right]^{-1 / 2}
$$

Pour notre cas de couplage

$$
\eta_{e}^{\prime} \approx \frac{\eta_{0}^{\prime}}{2}
$$

$P_{u}$ que nous appelons la puissance utile est la puissance $P_{2}$ (formule 3 ) où $G$ est remplacé par l'admittance de la cavité II, c'est la puissance d'excitation de la seconde cavité.

On reconnaît la relation donnant la sensibilité théorique d'un spectromètre utilisant un récepteur parfait, c'est-à-dire de facteur de bruit égal à 1 . C'est la sensibilité théorique que nous escomptions a priori. 
Néanmoins, puisque l'on connaît l'expression générale du signal (4) et l'expression générale du bruit (5), on peut déterminer l'absorption minimum détectable quel que soit le niveau d'oscillation et pour une fréquence de modulation quelconque.

Nous avons calculé pour notre cas le couplage, c'està-dire pour un $\eta_{e}^{\prime}$ donné et $Q_{0}^{\prime}$ donné, la valeur de $\chi_{\text {min }}^{\prime \prime}$ qui rend le rapport signal/bruit $=1$ pour une bande passante de détection de $1 / 10 \mathrm{~Hz}$.

La figure 7 montre le réseau obtenu : en abscisse sont portées les valeurs de $P_{1}$, c'est-à-dire le niveau

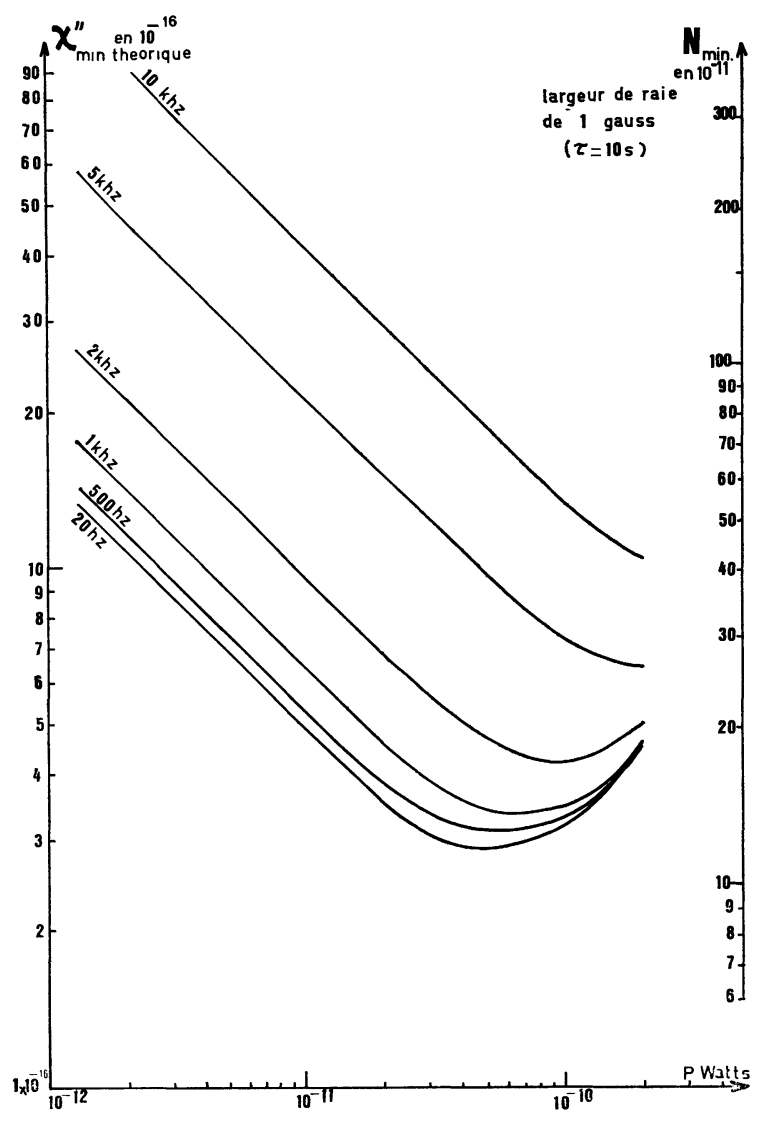

Fig. 7. - Sensibilité théorique du spectromètre en fonction du niveau et de la fréquence de modulation.

mesuré expérimentalement et en ordonnée la valeur de $\chi_{\min }^{\prime \prime}$ en $10^{-16}$ unités MKS. Les courbes en trait plein correspondent à différentes fréquences de modulation, au-dessous de $20 \mathrm{~Hz}$ les courbes se confondent avec celles à $20 \mathrm{~Hz}$. Il apparaît clairement que l'on a intérêt à employer une fréquence de modulation basse et que pour notre appareillage il existe une puissance optimale de fonctionnement qui est de l'ordre de $5 \times 10^{-11} \mathrm{~W}$; dans ce cas, le nombre minimum de centres détectables est de $10^{12}$.

L'échelle de droite donne le nombre minimum de centres détectables correspondant à l'échelle $\chi_{\min }^{\prime \prime}$ de gauche. Comme il est d'usage, le nombre de centres est ramené à une raie de largeur 1 G.
Pour tester la sensibilité expérimentale, nous avons utilisé un échantillon dont le nombre de centres étant connu, en employant une fréquence de modulation de $15 \mathrm{~Hz}$, le maser oscillant à un niveau tel que $P_{1}=10^{-11} \mathrm{~W}$.

La figure 8 montre l'enregistrement du signal : connaissant le rapport signal/bruit, on en déduit la

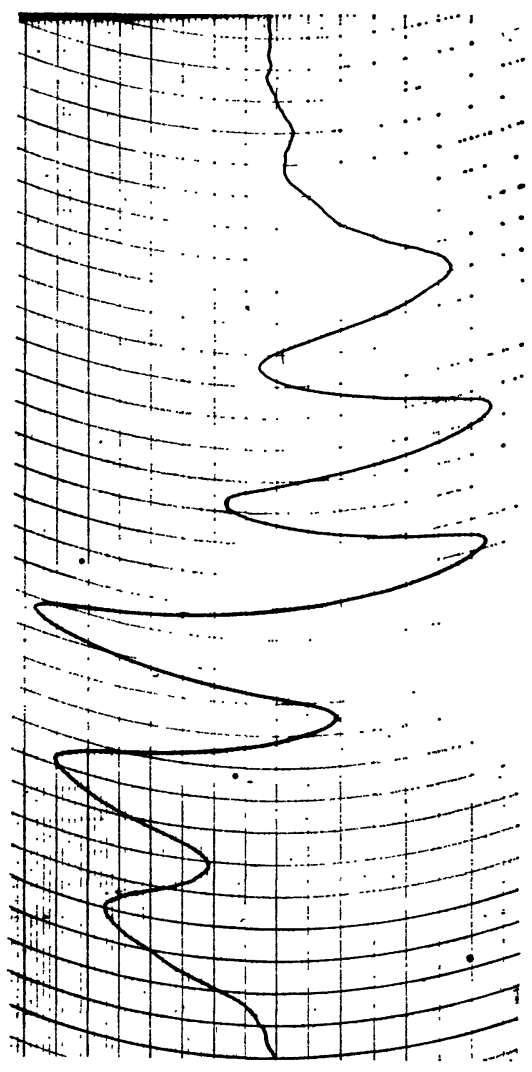

Fig. 8. - Signal d'absorption de $4 \times 10^{15}$ centres de D. P. P. H. (solution $\mathrm{M} / 1000) \tau=10 \mathrm{~s}$.

sensibilité expérimentale qui diffère de la sensibilité théorique calculée d'après les résultats précédents par un facteur de l'ordre de 2.

Conclusions. - La première est d'ordre instrumentale : nous avons réalisé un spectromètre autodyne - le premier à notre connaissance en ondes centimétriques - qui est aussi le seul à fonctionner avec une bonne sensibilité à un niveau d'excitation de la matière aussi faible que $10^{-12} \mathrm{~W}$. Ceci offre des perspectives intéressantes quant à l'étude des structures paramagnétiques à long temps de relaxation.

La seconde conclusion est que la sensibilité expérimentale approche la sensibilité théorique "idéale » obtenue en tenant compte du bruit de l'oscillateur.

La troisième est que le phénomène de R. P. E. qui permet de faire varier la charge d'un oscillateur de quantités connues, s'est révélé être un outil bien adapté à l'étude radioélectrique de l'oscillateur maser. 


\section{Bibliographie}

[1] Gambling (W. A.) and Wilmshurst (T. H.), $3^{\text {e conf. }}$ Electronique Quantique 1, III, 401, Dunod 1964.

[2] Townes (C. H.), Phys. Rev. Letters, 1960, 5, 428.

[3] HaRdin (J.), Thèse $3^{\mathrm{e}}$ cycle, Besançon, 1962.

[4] Pretot (R.), Thèse Université, Besançon, 1965.

[5] Slater (J.-C.), Rev. Mod. Phys., 1946, 18, 441.

[6] Shimoda (K.), Wang (T. C.), Townes (C. H.), Phys. Rev., 1956, 102, 1308.
[7] Hardin (J.), Thèse Doctorat, Besançon, 1970.

[8] Hardin (J.) et Uebersfeld (J.), C. $: R$. Acad. Sci. Paris, 1970, 270, 1617.

[9] HaAg (J.), Bull. Sc. Math., 1946.

[10] Balbi (J. H.) et Hardin (J.), C. R. Acad. Sci. Paris, 1971, 272, 413.

[11] Buyle Bodin (M.), J. Physique, 1959, 20, 159. 